\title{
Roncopatía primaria y sus implicancias clínicas: Fin al paradigma de entidad inocua
}

\author{
Primary snoring and clinical implications: End the harmless entity paradigm
}

\author{
Alfredo Santamaría C1, David Astudillo 0 1.
}

\begin{abstract}
RESUMEN
La Roncopatía Primaria constituye un trastorno respiratorio del sueño caracterizado por la ausencia de apneas, desaturaciones y microdespertares. Afecta a más del 50\% de la población adulta, traduciendo una obstrucción parcial de la vía aérea superior. Hasta hace poco, fue considerada por el mundo médico como una manifestación benigna sin un trasfondo patológico significativo, sin embargo, hoy sabemos que constituye una entidad patológica en sí misma y determina importantes repercusiones nocivas a nivel de funciones neurocognitivas, metabólicas y cardiovasculares. Si bien la polisomnografía persiste considerándose el gold standard en su estudio, nuevas líneas tales como análisis acústicos del ronquido presentan una relevancia creciente. En un futuro próximo, el estudio etiológico y de los mecanismos histopatológicos y bioquímicos que determinan las repercusiones clínicas de la roncopatía, aportará nuevas herramientas que contribuirán al desarrollo de una hoy necesaria nueva concepción de los trastornos obstructivos del sueño.
\end{abstract}

Palabras clave: Vía aérea superior, ronquido, roncopatía primaria.

\begin{abstract}
The primary snoring is a respiratory disorder of the sleep characterized by the absence of apnea, desaturation and arousals. It affects more than $50 \%$ of the adult population, exposing a partial obstruction of the upper airway. Until recently, it was considered by the medical world as a benign manifestation without significant pathological background, however, we now know that it is a disease entity in itself and determines important deleterious impact at neurocognitive, metabolic and cardiovascular levels. Although polysomnography still remains being the gold standard in its study, new lines such as acoustic analysis of snoring have an increasing relevance. In the near future, the study of the etiological, histopathological and biochemical mechanisms that determine the clinical implications of snoring, will provide new tools that will contribute to the development of a new conception of obstructive sleeping disorders.
\end{abstract}

Key words: Upper airway, snoring, primary snoring.

Médico. Departamento de Otorrinolaringologia, Universidad de Concepción. 


\section{GENERALIDADES}

La Roncopatía Primaria (RP) constituye uno de los Trastornos Respiratorio del Sueño (TRS), que por definición no se acompaña de apneas, hipopneas, desaturaciones ni microdespertares electroencefalográficos ${ }^{1}$.

Aun cuando el ronquido se presenta como una manifestación frecuente en la población, no constituye en caso alguno un evento fisiológico, por el contrario, su presencia pone en evidencia un grado variable de obstrucción de la vía aérea superior (VAS), variación anatómica que a su vez generará turbulencias en la columna aérea y la vibración de estructuras anatómicas, originando el sonido que clásicamente identificamos como ronquido, el que a su vez puede tener múltiples variaciones en concepto de tono e intensidad.

El ronquido, como síntoma, constituye el principal motivo de consulta entre quienes son portadores de un Síndrome de Apnea Obstructiva del Sueño (SAOS)2. Hasta hace apenas un par de décadas se aceptaba que en aquellas ocasiones en que el ronquido se presentaba en forma aislada, esto es, sin acompañarse de apneas o hipopneas, su presencia no se acompañaba de repercusión alguna sobre el estado de salud del paciente, constituyendo tan solo un problema social para su acompañante en la cama. En 1976, Guilleminault ${ }^{3}$ describió el Síndrome de Resistencia de la Vía Aérea Superior (SRVAS) en pacientes roncadores que si bien no presentan apneas ni hipopneas, generan altas presiones negativas a nivel de la vía aérea y el esófago, induciendo con ello microdespertares que determinan fragmentación del sueño y originan la hipersomnia diurna que aqueja a estos pacientes.

Durante la última década se ha publicado una serie de investigaciones que sustentan una nueva concepción de la RP, según la cual, esta entidad no tendría el carácter inocuo clásicamente descrito, sino por el contrario, condiciona un significativo deterioro de las funciones cognitivas y académicas de quienes la presentan, generando además alteraciones conductuales e incluso desórdenes metabólicos, especialmente en la población pediátrica ${ }^{4-10}$.

\section{EPIDEMIOLOGÍA}

Las corrientes de conocimiento que han conducido el estudio de los TRS y que son responsables de la mayor parte de la literatura científica disponible en este ámbito, han dado en general poca importancia clínica al ronquido, el que se ha tendido a considerar sólo un síntoma dentro del SAOS, o en su defecto, un síntoma que a futuro podría conducir a la aparición del SAOS, siendo esta la única traducción clínica hasta hace algunos años atribuible a la roncopatía.

Xiang, en un estudio que incluyó a 8.217 habitantes de Hong Kong ${ }^{11}$, describe una prevalencia de $59 \%$ en hombres y $47,9 \%$ en mujeres, dentro de una población adulta situada en el grupo etario entre 50 y 85 años. Por su parte, Nagayoshi refiere una prevalencia de $72,6 \%$ en hombres y $54,1 \%$ en mujeres para el grupo entre 40 y 69 años según datos de un estudio multicéntrico japonés ${ }^{12}$.

La prevalencia de Ronquido Habitual $(\mathrm{RH})$ en niños va desde cifras de 7,2\% según el estudio de Li en niños de 5 a 14 años en Hong Kong ${ }^{13}$, hasta $34,5 \%$ en el estudio de Castronovo en 595 niños de 2 a 8 años de una comunidad del norte de Ita$\mathrm{lia}^{14}$, siendo en este grupo, mayor la incidencia de $\mathrm{RH}$ en niños pequeños respecto a los mayores. La prevalencia de $34,5 \%$ citada fue obtenida mediante un cuestionario realizado a los padres, y se redujo hasta $23,4 \%$ en los 241 niños cuyos padres aceptaron completar el estudio mediante la realización de un monitoreo nocturno.

En general, para la población pediátrica se describe, al igual que entre los adultos, una mayor prevalencia de $\mathrm{RH}$ en el género masculino ${ }^{13-15}$. De igual manera, factores como obesidad, malformaciones craneofaciales y enfermedades neuromusculares se asocian también a una mayor prevalencia, alcanzando cifras que superan el $50 \%{ }^{16}$.

En nuestro país, Brockmann describe una prevalencia de $\mathrm{RH}$ en pacientes de 7 a 17 años del 18\% para una población urbana de nivel socioeconómico bajo ${ }^{15}$.

Sabemos que la obstrucción nasal puede favorecer la aparición de ronquido; este fenómeno se presenta frecuentemente como consecuencia de procesos infecciosos o inflamatorios, especialmente durante la edad pediátrica, sin embargo, su naturaleza transitoria y su extensión temporalmente limitada, determinan que no revista una mayor implicancia clínica. En este contexto, es necesario poner atención y dirigir nuestro estudio hacia el roncador habitual, cuyo ronquido puede evidenciar la presencia de una RP o bien constituir una manifes- 
tación más en el contexto de un SRVAS o un SAOS, el cual presenta una prevalencia 3,2 veces más alta en pacientes roncadores versus no roncadores ${ }^{17}$.

\section{FISIOPATOLOGÍA}

En términos prácticos, podemos definir ronquido como la emisión de respiración ruidosa durante el sueño, con una intensidad mayor a $50 \mathrm{~dB}$ cuando es medida cerca del sitio de emisión, esto es, a nivel del labio superior ${ }^{18}$.

Esta respiración ruidosa se origina producto del colapso parcial de ciertas regiones anatómicas que componen la arquitectura de la vía aérea superior. Este colapso se ve favorecido por el estrechamiento luminal de la vía aérea, producto principalmente de variaciones constitucionales que condicionan un mayor volumen de determinadas estructuras (hiperplasia adenoidea $y / 0$ amigdalina, hipertrofia de la base lingual, elongación y engrosamiento del paladar blando, entre otros). El aumento de la velocidad y la turbulencia producida en la columna áerea como consecuencia de este estrechamiento, condicionan la vibración de estructuras anatómicas y con ello el origen del ronquido.

La disminución del tono muscular durante el sueño también juega un rol relevante en su génesis, es así que los TRS aumentan su incidencia durante la etapa de sueño REM ${ }^{1}$.

Durante mucho tiempo, se aceptó que la obstrucción de la VAS se producía de manera casi exclusiva durante la inspiración, puesto que durante esta fase se genera una presión negativa intraluminal que propicia el colapso de estructuras no rígidas de la VAS, tales como paladar blando, base de lengua y supraglotis. No obstante este precepto, algunos estudios experimentales demestran la producción de colapsos espiratorios con relevancia clínica significativa en pacientes con RP y SAOS ${ }^{1}$.

La intensidad del ronquido dependería del grado de obstrucción y la diferencia de presiones generada ${ }^{19}$. En este marco, debemos considerar que la obstrucción nasal, ya sea por inflamación, hipertrofia de cornetes, poliposis nasal 0 desviaciones septales, al condicionar un aumento en la presión negativa inspiratoria, favorece tanto la aparición de ronquidos, como la acentuación de un cuadro de RP o SAOS.
La conformación cráneo facial y del cuello de los individuos, influye en la posibilidad de presentar ronquido o SAOS; estudios cefalométricos en grupos de pacientes sanos, roncadores y con SAOS, presentan medidas significativamente distintas en sus diferentes estructuras ${ }^{20}$. Así pues, el sólo hecho de presentar un perímetro cervical superior a $43 \mathrm{~cm}$ constituye por sí solo un factor de riesgo para roncar y presentar SAOS ${ }^{21}$.

\section{IMPLICANCIAS NEUROCOGNITIVAS}

Las repercusiones metabólicas, sociales y neurocognitivas en pacientes portadores de SAOS han sido aceptadas y estudiadas ampliamente, no así las producidas en pacientes portadores de RP, la que hasta hace poco se consideraba una entidad inocua 0 una simple manifestación de un SAOS leve. En los últimos años, un número creciente de literatura científica abarca y profundiza el entendimiento de estos aspectos, especialmente en la población infantojuvenil.

En escolares, la incidencia de RP es alrededor de $12 \%$, mientras que $1 \%$ a $2 \%$ presentaría un SAOS. Gozal ${ }^{5}$ estudió a 297 escolares de primer año que se encontraban en el percentil 10 de menor rendimiento académico, describiendo una alta incidencia $(18,1 \%)$ de probable SAOS de acuerdo a la aplicación de una encuesta a los padres y mediciones de oximetría y capnografía nocturna.

Brockmann ${ }^{4}$ estudió el deterioro neurocognitivo en 1.114 escolares; realizando estudio con polisomnografía (PSG) a los individuos roncadores, a fin de identificar y discriminar entre quienes presentaban RP, SAOS y SRVAS; demostró que el grupo de RP presenta un deterioro similar al identificado en los grupos con SAOS y SRVAS en cuanto a hiperactividad, déficit atencional, hipersomnia diurna, rendimiento académico en matemáticas, ciencias y lenguaje (ver Tabla 1).

El mismo autor, en un estudio realizado a niños chilenos entre 7 y 17 años, en un entorno de población urbana de nivel socioeconómico bajo, encontró una alta tasa de Trastornos Respiratorios del Sueño (TRS), que se asociaron en forma significativa a comportamiento hiperactivo y un bajo rendimiento académico ${ }^{15}$.

Bourke, en dos estudios ${ }^{6,10}$, realizó PSG a niños de 7 a 12 años con desórdenes obstructivos 
Tabla 1. Síntomas diurnos y rendimiento escolar: Estratificación según trastorno respiratorio del sueño

\begin{tabular}{|l|c|c|c|c|}
\hline & $\begin{array}{c}\text { NR } \\
\text { (N 410) }\end{array}$ & $\begin{array}{c}\text { RP } \\
\text { (N 69) }\end{array}$ & $\begin{array}{c}\text { SRVAS/SAOS } \\
\text { (N 23) }\end{array}$ & $p$ \\
\hline Hiperactividad, desatención $^{\mathrm{a}}$ & 1,5 & 2,4 & 2,0 & $<0,0001$ \\
\hline Hipersomnia diurna $^{\mathrm{a}}$ & 0,6 & 1,3 & 1,2 & $<0,0001$ \\
\hline $\begin{array}{l}\text { Rendimiento escolar: } \\
\text { Matemáticas }^{\mathrm{b}}\end{array}$ & 70,8 & 58,8 & 58,6 & 0,002 \\
\hline Rendimiento escolar: Ciencia $^{\mathrm{b}}$ & 71,6 & 64,3 & 61,0 & 0,041 \\
\hline $\begin{array}{l}\text { Rendimiento escolar: } \\
\text { Lenguajec }^{\mathrm{b}}\end{array}$ & 72,6 & 66,5 & 57,6 & 0,017 \\
\hline
\end{tabular}

a Score numérico asignado arbitrariamente: 0 (nunca), 1 (ocasionalmente), 2 (casi siempre).

${ }^{b}$ Percentil de rendimiento escolar respecto a sus pares.

Pablo E. Brockmann, Michael S. Urschitz, Martin Schlaud, Christian F. Poets. Primary snoring in school children: prevalence and neurocognitive impairments. Sleep Breath 2012; 16: 23-9.

del sueño, y a un grupo control de niños sanos. Demostró que los niños con RP, SAOS leve y SAOS moderado/severo presentan un deterioro significativo en las funciones cognitivas (habilidades ejecutivas e intelectuales) y académicas, en relación al grupo control, independiente de la severidad del cuadro, teniendo los portadores de RP (sin hipoxias ni fragmentación del sueño) similar deterioro que aquellos con diagnóstico de SAOS.

Respecto a las alteraciones en la memoria de trabajo, estudios que abarcan de manera específica este tópico evidencian resultados contradictorios según el método de evaluación aplicado; al emplear evaluaciones subjetivas como la apreciación de los padres (Behaviour Rating Inventory of Executive Function, BRIEF), los resultados son peores para los portadores de SAOS y RP versus pacientes sanos, sin embargo, al utilizar métodos de medición más objetivos, como el reconocimiento por computador de cartas de juego (CogHealth), no se aprecian diferencias significativas con respecto a los grupos control ${ }^{8}$.

A la luz de estas experiencias, podemos concluir con bases sólidas, que la RP sin desaturaciones, apneas ni hipopneas, no es tan inocente como hasta hace algunos años se aceptaba y que, por el contrario, produce un impacto en el comportamiento, así como en el desarrollo cognitivo y emocional del niño.

\section{IMPLICANCIAS METABÓLICAS Y CARDIOVASCULARES}

$\mathrm{Su}^{22}$ estudió la relación entre RP, SAOS, síndrome metabólico (SM) y niveles sanguíneos de PCR ultrasensible (usPCR), un marcador indirecto de alto riesgo cardiovascular. Se analizaron 309 pacientes con TRS. En general, niveles elevados de usPCR se asociaron a alteraciones metabólicas y a un mayor riesgo cardiovascular, independiente de la severidad del TRS, esto es, sin diferencias significativas entre RP y SAOS.

En esta misma línea, Kazman ${ }^{23}$ estudió a 248 adultos afroamericanos de Maryland, describiendo una correlación estadísticamente significativa entre la presencia de ronquido y el diagnóstico de síndrome metabólico, así como también con alteraciones específicas en factores de riesgo cardiovascular tales como alteración de glicemia en ayunas, IMC, porcentaje de masa corporal y circunferencia de cintura.

EI SAOS origina disfunción endotelial en adultos y niños ${ }^{24-26}$. Li estudió 201 niños de 6 a 18 años para determinar el grado de función endotelial utilizando ultrasonografía de vasodilatación mediada por el flujo de la arteria braquial, método considerado el gold standard para esta medición'; demostró que los niños con RP presentan una disfunción endotelial significativa en relación a los no roncadores, independiente de si presentan un 
peso normal o sobrepeso $0^{9}$. La disfunción endotelial constituye el indicador más precoz de patología cardiovascular y su presencia es altamente predictiva de eventos cardiovasculares en personas durante las primeras etapas de la enfermedad, independiente de los factores de riesgo convencionales ${ }^{27,28}$.

Los pacientes portadores de TRS presentan también una alteración en la autorregulación autómica, lo que condiciona un aumento de la presión arterial y la frecuencia cardiaca, tanto en adultos ${ }^{29}$ como en la población pediátrica ${ }^{7}$. La Tabla 2 expone que si bien quienes tenían RP no presentan alteraciones hemodinámicas estadísticamente significativas, mayores respecto al grupo control, sí existe una tendencia en este sentido.

Respecto a la etiología de estas alteraciones, se postula que periodos repetidos de hipoxia y reoxigenación, excitación del sistema nervioso central y fluctuaciones de la presión intratorácica, son los principales mecanismos responsables de la comorbilidad cardiovascular en los TRS. Estos fenómenos conducen a un aumento del estrés oxidativo, inflamación sistémica crónica, trastorno de la función vasomotora, incremento de la actividad simpática y desarrollo de arteriosclerosis ${ }^{29}$.

\section{ESTUDIO Y DIAGNÓSTICO}

La observación por un testigo de las características del ronquido, asociado a un sueño reparador y la ausencia de hipersomnia diurna, constituyen en conjunto, elementos suficientes para establecer el diagnóstico de RP. No obstante, la práctica clínica nos ha enseñado que un paciente con estas características, tanto más un individuo joven, puede ya encontrarse desarrollando un SAOS o un SRVAS, sin dar manifestaciones tan evidentes de ello.

En un intento por implementar un método de screening entre pacientes roncadores susceptibles de presentar un TRS de mayor complejidad, encubierto 0 aún no develado, hay quienes postulan la realización de un análisis acústico del ronquido, empleando para esto un Programa Multidimensional de la Voz, el cual analiza variadas características del ronquido. Mediante la aplicación de este método, se ha logrado demostrar diferencias significativas entre los peaks de intensidad y el espectro frecuencial de pacientes portadores de RP y SAOS, llegando incluso a poder clasificar a los pacientes en grupos de severidad dependiendo del valor esperado del IAH',17,30. Estos estudios, si bien son prometedores, aún necesitan una mayor validación para ser aplicados en la práctica clínica diaria.

Tabla 2. Presión arterial y frecuencia cardiaca en niños sanos, con RP y SAOS, durante periodos de vigilia y en las distintas etapas del sueño

\begin{tabular}{|l|c|c|c|c|}
\hline & Control & RP & SAOS leve & $\begin{array}{c}\text { SAOS } \\
\text { moderado/severo }\end{array}$ \\
\hline Presión arterial (mmHg) & \multicolumn{3}{|l|}{} \\
\hline Despierto & $62 \pm 2$ & $71 \pm 3^{*}$ & $69 \pm 2^{*}$ & $75 \pm 3^{*}$ \\
No REM & $62 \pm 2$ & $67 \pm 2^{*}$ & $69 \pm 2^{*}$ & $74 \pm 2^{*}$ \\
Sueño onda lenta & $60 \pm 1$ & $63 \pm 2$ & $69 \pm 3^{*}$ & $67 \pm 3^{*}$ \\
REM & $68 \pm 2$ & $72 \pm 2$ & $78 \pm 3^{*}$ & $80 \pm 3^{*}$ \\
\hline Frecuencia cardiaca (Ipm) & \multicolumn{5}{|l|}{} \\
\hline Despierto & $83 \pm 2$ & $85 \pm 2$ & $85 \pm 2$ & $92 \pm 2^{*}$ \\
No REM & $70 \pm 1$ & $74 \pm 2$ & $74 \pm 2$ & $80 \pm 2^{*}$ \\
Sueño onda lenta & $74 \pm 1$ & $75 \pm 2$ & $75 \pm 2$ & $83 \pm 1^{*}$ \\
REM & $74 \pm 1$ & $76 \pm 1$ & $78 \pm 2$ & $84 \pm 2^{*}$ \\
\hline
\end{tabular}

${ }^{*} p<0,05$ en relación al grupo control.

Lisa M. Walter, Gillian M. Nixon, Margot J. Davey, Vicki Anderson \& Adrian M. Walker, Rosemary S. C. Horne. Autonomic dysfunction in children with sleep disordered breathing. Sleep Breath; Published online 09 June 2012. 
Darre $\left.\right|^{18}$ en un estudio domiciliario, mediante mediciones de la intensidad del ronquido, demostró una asociación significativa entre la duración del ronquido, su intensidad promedio y el peak de intensidad, respecto al índice de masa corporal (IMC) y el IAH; de igual manera, describió una asociación entre la intensidad promedio y la escala de Epworth (ESDE). Según Darrel, existe una progresión lineal de $20 \mathrm{~dB}$ entre RP y SAOS severo y la duración del ronquido aumenta desde $73 \%$ en $\mathrm{RPa}$ $98 \%$ en SAOS severo. Con estas consideraciones, cada vez que el ronquido tiende a presentarse de manera frecuente y con cierto nivel de intensidad, se recomienda realizar un estudio de sueño, polisomnografia (PSG) o poligrafía ambulatoria del sueño (PAS).

Hunsaker realizó una evaluación domiciliaria de pacientes que presentaban TRS, incorporando a los métodos de estudio convencionales, la medición de la intensidad del ronquido. Sus resultados sugieren que la RP constituye un factor independiente de disrupción del sueño ${ }^{18}$, esto, puesto que existe un subgrupo de pacientes en quienes el Índice de Apnea e Hipopnea (IAH) es normal, no obstante, roncan y presentan síntomas de hipersomnia diurna ${ }^{18}$; según Hunsaker, la intensidad del ronquido estaría relacionada con el nivel de disrupción del sueño y la afectación del acompañante en la cama, por lo que esta medición debiera ser incorporada en los estudios del sueño de manera habitual.

Los métodos de screening automáticos mencionados, tienen a su favor el hecho de ser bastante más sencillos y económicos que la realización de una PSG, por lo que su implementación podría permitirnos seleccionar aquellos pacientes de mayor riesgo, en quienes finalmente realizar una PSG, examen costoso y cuya realización resulta compleja para el paciente, tanto más en edad pediátrica.

La necesidad del estudio polisomnográfico en niños con SAOS es controversial, ya que la mayoría de las veces esta patología se origina por una hiperplasia adenoamigdaliana y se resuelve fácil y exitosamente mediante una adenoamigdalectomía; en general, por lo complejo y costoso que resulta la realización de una PSG en edad pediátrica, se prefiere efectuarla sólo encasos seleccionados, tales como portadores de malformaciones cráneo faciales, síndromes hipotónicos 0 en quienes el examen físico no arroja hallazgos consistentes con el diagnóstico. Por su parte, existe acuerdo de realizar una PSG en niños que fueron intervenidos quirúrgicamente, pese a lo cual las apneas continúan. En el caso de la RP con examen acorde a la sintomatología, sin otras comorbilidades importantes, no se justificaría una PSG.

Akpinar estudió las características cefalométricas en pacientes adultos de sexo masculino no obesos ${ }^{20}$, demostró mediante este estudio que el área retropalatal, la distancia entre la mandíbula y el hioides, así como la longitud y el espesor del paladar blando, presentan diferencias significativas entre individuos sanos y aquellos con diagnóstico de RP y SAOS, proponiéndose que los datos cefalométricos seleccionados pueden ser útiles al momento de determinar el sitio anatómico de obstrucción de la VAS, constituyendo un complemento significativo a la realización de estudios endoscópicos y la PSG.

\section{TRATAMIENTO}

El uso de aparatos de Presión Positiva de Vía Aérea (PAP), ha demostrado ser muy eficiente en inhibir la aparición del ronquido, no obstante, pacientes que presentan únicamente RP, sin SAOS, especialmente sujetos jóvenes, tienen una baja tasa de adherencia a su uso. Por otra parte, muchos autores consideran excesiva la indicación de PAP en RP, en condiciones en las que esta patología puede muchas veces manejarse satisfactoriamente de forma conservadora mediante la optimización del estado ponderal, mediadas de higiene del sueño y manejo posicional.

Aarts ${ }^{31}$ evaluó la respuesta al uso de Dispositivos Orales (D0) en el manejo de la RP, encontrando $38 \%$ de reducción en la sonoridad del ronquido y $54 \%$ de mejoría en los trastornos del sueño del compañero de cama. Por su parte, Maguire ${ }^{32}$ describió una mejoría significativa mediante el uso tanto de dispositivos de avance mandibular, como de dispositivos de elevación de la mordida.

El rol de la obstrucción nasal no está aún totalmente aclarado en la fisiopatología del ronquido y el SAOS, sin embargo, pacientes portadores de SAOS presentan una alta tasa de anormalidades obstructivas a nivel nasal, por lo que se intuye que debiera existir algún grado de influencia en 
su etiopatogenia ${ }^{33}$. Al respecto, Craig ${ }^{34}$ revisó tres estudios con uso de corticoides tópicos versus grupos control con placebo en pacientes portadores de rinitis alérgica, los cuales demostraron que el uso de esteroides intranasales como tratamiento único, mejoraba la calidad del sueño, disminuía en forma significativa la congestión nasal, los TRS y la hipersomnia diurna. No está claro si estos cambios se deben sólo a un efecto mecánico producto de la corrección de la obstrucción nasal, o si bien juega un papel preponderante el efecto antiinflamatorio producto de la disminución de citokinas proinflamatorias tales como IFN-gama, TNF-alfa, IL-1B, IL-4 e IL 10.

El efecto de la cirugía para mejorar la permeabilidad nasal en SAOS es controversial, ya que si bien la cirugía (septoplastía, turbinoplastía, polipectomías, entre otros) disminuye la sintomatología en cuanto a mejorar la obstrucción nasal, reducir el ronquido, disminuir la hipersomnia y minimizar los efectos negativos sobre el acompañante en la cama, el estudio polisomnográfico de control posoperatorio evidencia una mantención e incluso un incremento en los valores de IAH en un significativo porcentaje de estos pacientes ${ }^{33,35}$.

Es importante no dejar de lado el hecho de que la obstrucción nasal puede ser un obstáculo en la adaptación de un dispositivo oral, así como puede también obligar al usuario de PAP a recurrir al empleo de altas presiones, lo que reduce la adherencia a su uso. En este contexto, la cirugía nasal tiene un rol positivo como parte del tratamiento de estos pacientes ${ }^{33,35}$.

La uvulopalatofaringoplastía (UPFP) fue descrita inicialmente en 1964 por Ikematsu en Japón ${ }^{36}$ y posteriormente introducida a Estados Unidos en el año 1981 por Fujita ${ }^{37}$, para el tratamiento quirúrgico del SAOS y la roncopatía; existen en la actualidad múltiples variaciones de la técnica, teniendo en común modestos resultados en términos de tratamiento exitoso del SAOS; no obstante, esto se debe primordialmente a una inadecuada indicación y selección de pacientes. La Tabla 3 expone resultados quirúrgicos utilizando UPFP como tratamiento único, según la etapificación de Fried$\operatorname{man}^{38}$, en la que los estadíos bajos corresponden a pacientes con amígdalas grandes y base de lengua pequeña, y los estadíos altos viceversa. Teniendo en cuenta estos resultados, podemos concluir que mediante una adecuada selección de pacientes, se pueden efectivamente alcanzar buenos resultados mediante la implementación de esta técnica en el tratamiento del SAOS. No obstante lo expuesto, la UPFP es considerada por algunos autores como una cirugía demasiado agresiva para el tratamiento de una RP aislada.

En niños normales, gran parte de la roncopatía habitual se origina de una hiperplasia adenoidea y/o amigdalina, siendo menos frecuente la hipertrofia de cornetes, la rinitis crónica y las desviaciones septales. En este contexto, la adenoamigdalectomía, por tratarse de una cirugía rápida, de relativo bajo costo y de pronta recuperación en los niños, es el tratamiento lógico para quienes presentan un TRS causado por la hiperplasia de estos órganos linfoides.

Como alternativa quirúrgica en adultos, ante otras etiologías, existe lo que conocemos como procedimientos mínimos, usados con el objetivo de incrementar la rigidez del velo del paladar, disminuyendo su capacidad vibrátil durante el sueño, con la consiguiente reducción del ronquido; entre

Tabla 3. Tasa de éxito de la UPFP, utilizada como tratamiento único en el manejo de los TRS. Selección del paciente quirúrgico según clasificación de Friedman

\begin{tabular}{|c|c|c|c|}
\hline Estadío & Fracaso & Éxito & Total \\
\hline I & $6(19,4 \%)$ & $25(80,6 \%)$ & $31(100 \%)$ \\
\hline II & $18(62,1 \%)$ & $11(37,9 \%)$ & $29(100 \%)$ \\
\hline III & $68(91,9 \%)$ & $6(8,1 \%)$ & $74(100 \%)$ \\
\hline
\end{tabular}

Michael Friedman. Sleep apnea and snoring: surgical and non-surgical therapy. Editorial Saunders, Elsevier, 2009. 
los más usados están la inyección de sustancias esclerosantes y la cauterización con radiofrecuencia, esta última provoca una quemadura de baja temperatura en el espesor de la musculatura del paladar, generando una reacción inflamatoria que conduce a la fibrosis a partir del primer mes poscirugía. También puede emplearse la colocación de implantes en los músculos palatinos, lo que induce también una reacción inflamatoria, con la consiguiente fibrosis y rigidez del velo. Todos estos procedimientos pueden ser realizados en forma ambulatoria y bajo anestesia local, con una baja tasa de complicaciones ${ }^{38}$. En este caso, los resultados para RP a corto y mediano plazo son aceptables, sin embargo, su efectividad es cuestionable cuando se evalúan a largo plazo ${ }^{38,39}$.

En los años 90 se preconizó el uso del láser $\mathrm{CO}_{2}$ como alternativa para el manejo de la roncopatía y el SAOS, realizándose diferentes técnicas de resección y vaporización de tejidos faríngeos, palatinos y amigdalinos ${ }^{38}$. Los resultados a largo plazo han sido decepcionantes, por no mencionar lo doloroso del periodo posoperatorio y el porcentaje significativo de complicaciones reportadas, tales como estenosis faríngeas e insuficiencia velofaríngea, situaciones difíciles de corregir. Por lo anterior, su uso tiene actualmente pocos adeptos.

\section{DISCUSIÓN}

Como primer y principal concepto, nos parece imperioso rescatar y establecer el hecho de que la $\mathrm{RP}$, por sí misma, constituye una entidad patológica con repercusiones clínicas importantes, y de perpetuarse en el tiempo, eventualmente graves, tanto para el paciente que lo padece, como para su compañero de cama. Estas repercusiones adquieren mayor trascendencia por cuanto se presentan desde la infancia, demostrándose ya alteraciones anatómicas, fisiológicas, bioquímicas y conductuales durante la edad escolar; lo que a nuestro entender, debiera condicionar, no sólo un cambio en el paradigma de enfrentamiento de la RP, sino también en el enfrentamiento preventivo de la patología cardiovascular.

Los mecanismos exactos por los cuales la RP genera un deterioro cognitivo, académico y metabólico no están aún aclarados; desde el punto de vista metabólico, un estudio experimental en animales demostró que la estimulación con intensos sonidos, causando vibración traqueal y del velo palatino durante tres horas, indujo una significativa sobreexpresión de biomarcadores proinflamatorios, tales como TNF alfa y proteína inflamatoria 2 macrofágica ${ }^{40}$; por tanto, la vibración de los tejidos faríngeos pudiera constituir el factor gravitante que desencadena la liberación de citoquinas proinflamatorias causantes de los efectos metabólicos nocivos descritos en RP.

En lo que respecta al estudio de esta entidad, una posibilidad es que los actuales estudios PSG no sean lo suficientemente sensibles en detectar cambios mínimos producidos en los parámetros cardiovasculares, de oxigenación y arquitectura del sueño eventualmente producidos por la RP. Otro factor relevante a considerar, es el hecho de que en gran parte de los estudios científicos disponibles, se realizó un monitoreo con PSG durante sólo una noche, lo que pudiera resultar insuficiente para analizar una afección crónica que fomenta su fisiopatología precisamente en la exposición reiterada de la vía aérea a condiciones adversas de trauma, inflamación e hipooxigenación. En los estudios realizados con PSG en RP no se hace un análisis de las posibles alteraciones de la arquitectura del sueño, en relación al porcentaje de duración de las diferentes etapas de éste.

El estudio de la RP como entidad clínica patológica y no sólo como manifestación de un cuadro más complejo, plantea múltiples interrogantes, a la vez que expone nuevas áreas de investigación hasta ahora inexploradas, pero cuyo conocimiento tendrá sin duda repercusiones importantes en la neurología, otorrinolaringología, la endocrinología y la cardiología, entre otras áreas del desarrollo médico.

\section{BIBLIOGRAFÍA}

1. B TuCKer Woodson. Expiratory Pharyngeal Airway Obstruction During Sleep: A Multiple Element Model. Laryngoscope 2003; 113: 1450-9.

2. Hirotaka Hara, Naoko Murakami, Yuj Miyauchi, HIROSHI Yamashita. Acoustic Analysis of Snoring Sounds by aMultidimensional Voice Program. Laryngoscope 2006; 116: 379-81. 
3. Guilleminault C, Tilkian A, Eldridge F, Simmons F, Dement W. Sleep apnea syndrome due to upper airway obstruction: a review of 25 cases. Arch Intern Med 1976; 137: 296-300.

4. Pablo E. Brockmann, Michael S. Urschitz, Martin Schlaud, Christian F. Poets. Primary snoring in school children: prevalence. and neurocognitive impairments. Sleep Breath, Published online 16 January 2011.

5. DaVID Gozal. Sleep-Disordered Breathing and School Performance in Children. Pediatrics 1998; 102: 616.

6. Robert S. Bourke, Vicki Anderson, Joel SC Yang, ET COLS. Neurobehavioral function is impaired in children with all severities of sleepdisordered breathing. Sleep Medicine 2011; 12: 222-9.

7. Lisa M. Walter, Gillian M. Nixon, Margot J. Davey, Vicki Anderson, Adrian M. Walker, Rosemary S. C Horne. Autonomic dysfunction in children with sleep disordered breathing. Sleep Breath, Published online 09 June 2012.

8. Sarah N. Biggs, Robert Bourke, Vicki Anderson, y cols. Working memory in children with sleep-disordered breathing: Objective versus subjective measures. Sleep Medicine 2011; 12 : 887-91.

9. Albert M. Li, Chun T. Au, P. Chook, Hugh S. Lam, Yun K. WING. Reduced flow-mediated vasodilation of brachial artery in children with primary snoring. International Journal of Cardiology 2012.

10. Robert Bourke, Vicki Anderson, Joel S.C. Yang, ET COLS. Cognitive and academic functions are impaired in children with all severities of sleepdisordered breathing. Sleep Medicine 2011; 12 : 489-96.

11 Xiang Qian Lao, G. Neil Thomas, Chao Qiang Jiang, ET CoLs. Obesity, high-sensitive C-reactive proteinand snoring in older Chinese: The Guangzhou Biobank Cohort Study. Respiratory Medicine 2010; 104: 1750-6.

12. Mako Nagayoshi, Takeshi Tanigawa, Kazumasa Yamagishi, ET coLs. Habitual Snoring and Cardiovascular Events. J Epidemiol 2012; 22(4): 295-301.

13. Albert M. Li, Chun T. Au, Hung K. So, Joseph Lau, PaK C. Ng, Yun K. Wing. Prevalence and Risk Factors of Habitual Snoring in Primary School Children. Chest 2010; 138: 519-27.

14. Vincenza Castronovo, Marco Zucconi, Luana Nosetti, Y cols. Prevalence of Habitual Snoring and
Sleep-Disordered Breathing in Preschool-Aged Children in an Italian Community. The Journal of Pediatrics 2003; 377-83.

15. Brockmann PE, Bertrand P, Pardo T, Cerda J, Reyes B, Holmgren NL. Prevalence of habitual snoring and associated neurocognitive consequences among Chilean school aged children. International Journal of Pediatric Otorhinolaryngology 2012; 76 (9): 1327-31.

16. Friedman, Michael. Sleep apnea and snoring: surgical an son-surgical therapy; Saunders Elseviere, 2009.

17. Jordi SolÀ-Solera, José Antonio Fiz, José Morerac, RAIMON JanÉA. Multiclass classification of subjects with sleep apnoea-hypopnoea syndrome through snoring analysis. Medical Engineering \& Physics 2012; 34: 1213-20.

18. Darrell H. Hunsaker, Robert H. Riffenburgh. Snoring significance in patients undergoing homesleep studies. Otolaryngology-Head and Neck Surgery 2006; 134: 756-60.

19. Pevernagie D, Aarts RM, De Meyer M. The acoustics of snoring. Sleep Med Rev 2010; 14(2): 131-44.

20. Meltem Esen Akpinar, M. Mazhar Celikoyar, Aytug Altundag, Ismail Kocak. The comparison of cephalometric characteristics in non obese obstructive sleep apnea subjects and primary snorers cephalometric measures in non obese OSA and primary snorers. Eur Arch Otorhinolaryngol 2011; 268: 1053-9.

21. Iriondo Bedialauneta JR, Santaolalla Montoya $F$, Moreno Alonso E, Martínez Ibargüen A, Sánchez Fernández JM. Analysis of the anthropometric, epidemiological, and clinical parameters in patients with snoring and obstructive sleep apnoea. Acta Otorrinolaringol Esp 2007; 58(9): 413-20.

22. Su MC, Chen YC, Huang KT, Wang CC, Lin MC, LIN HC. Association of metabolic factors with high-sensitivity C-reactive protein in patients with sleep-disordered breathing. Eur Arch Otorhinolaryngol 2012 Sep 28.

23. Kazman JB, Abraham PA, Zeno SA, Poth M, Deuster $P A$. Self-reported sleep impairment and the metabolic syndrome among African Americans. Ethn Dis 2012; 22(4): 410-5.

24. David Gozal, Leila Kheirandish-Gozal, Rakesh Bhattacharjee, Karen Spruyt. Neurocognitive 
and Endothelial Dysfunction in Children With Obstructive Sleep Apnea. Pediatrics 2010; 126: 1161.

25. Rakesh Bhattacharjee, Wadha H Alotaibi, Leila Kheirandish-Gozal, Oscar Sans Capdevila, David Gozal. Endothelial dysfunction in obesenon-hypertensive children without evidence ofsleep disordered breathing. BMC Pediatrics 2010; 10: 8.

26. Leila Kheirandish-Gozal, Rakesh Bhattacharjee, David Gozal. Autonomic alterations and endothelial dysfunction in pediatric obstructive sleep apnea. Sleep Medicine 2010; 11: 714-20.

27. Celermajer DS, Sorensen KE, Gooch VM, et al. Non invasive detection of endothelial dysfunction in children and adults at risk of atherosclerosis. Lancet 1992; 340: 1111-5.

28. Chan Sy, Mancinı GB, Kuramoto L, Schulzer M, Frohlich J, Ignaszewski A. The prognostic importance of endothelial dysfunction and carotid atheroma burdenin patients with coronary artery disease. J Am Coll Cardiol 2003; 42: 1037-43.

29. Meier M, Andreas S. Mechanisms of cardiovascular co-morbidity in patients with obstructive sleep apnoea syndrome. Pneumologie 2012; 66(11): 650-7.

30. José Antonio Fiz, Raimon Jane, Jordi Sola-Soler, Jorge Abad, M. Angeles García, José Morera. Continuous Analysis and Monitoring ofSnores and Their Relationship to the Apnea-Hypopnea Index. Laryngoscope 2010; 120: 854-62.

31. Aarts MC, Rovers MM, Van der HeiJden GJ, Grolman $W$. The value of a mandibular repositioning appliance for the treatment of non apneic snoring. Otolaryngol Head Neck Surg 2011; 144(2): 170-3.
32. Maguire J, Steele JG, Gibson GJ, Wilson JA, Steen N, McCracken Gl. Randomised cross-over study of oral appliances for snoring. Clin Otolaryngol 2010; 35: 204-9.

33. Carolina Cozzi Machado, Fernanda Louise Martinho HADDAD. The role of the nose in obstructive sleep apnea. Sleep Sci 2012; 5(2): 61-4.

34. Timothy J. Craig, Christopher D. Hanks, Laura H. Fisher. How do topical nasal corticosteroidsimprove sleep and daytime somnolencein allergic rhinitis? J Allergy Clin Immunol 2005; 116 (6): 1264-6.

35. Michael Friedman, Zubair Syed, Roee Landsberg. The role of nasal obstruction and nasal surgery in the pathogenesis and treatment of obstructive sleep apnea and snoring. Curr Opin Otolaryngol Head Neck Surg 2001; 9: 158-61.

36. IKEMATSU T. Study of snoring $4^{\text {th }}$ report Therapy. $J$ Jpn Otol Rhinol Layngol Soc 1964; 64: 434-5.

37. Fujita S, Conway W, Zorick F, Roth T. Surgical correction of anatomic abnormalities in obstructive sleep apnea syndrome: uvulopalatopharyngoplasty. Otolaryngol Head Neck Surg 1981; 89: 923-34.

38. Michael Friedman. Sleep apnea and snoring: surgical and non-surgical therapy. Editorial Saunders, Elsevier, 2009.

39. Brian W. Rotenberg, Kimberly Luu. Four-Year Outcomes of Palatal Implants for Primary Snoring Treatment: A Prospective Longitudinal Study. Laryngoscope 2012; 122: 696-9.

40. Almendros I, Acerbi I, Puig F, Montserrat JM, Navajas D, FarRÉ R. Upper-airway inflammation triggered by vibration in a rat model of snoring. Sleep 2007; 30: 225-7. 